\title{
1-Methyl-1,2,3,4-Tetrahydroisoquinoline, an Endogenous Amine with Unexpected Mechanism of Action: New Vistas of Therapeutic Application
}

\author{
Lucyna Antkiewicz-Michaluk • Agnieszka Wąsik • \\ Jerzy Michaluk
}

Received: 4 April 2013/Accepted: 6 May 2013/Published online: 30 May 2013

(c) The Author(s) 2013. This article is published with open access at Springerlink.com

\begin{abstract}
This review outlines the effects of 1,2,3,4-tetrahydroisoquinoline (TIQ) and its derivative, 1-methyl1,2,3,4-tetrahydroisoquinoline (1MeTIQ), endogenous substances imbued with high pharmacological potential and broad spectrum of action in brain. 1MeTIQ has gained special interest as a neuroprotectant, and its ability to antagonize the behavioral syndrome produced by well-known neurotoxins (e.g., MPTP; rotenone). This review is thus focused on mechanisms of action of 1MeTIQ in behavioral, neurochemical, and molecular studies in rodents; also, effects of TIQ and 1MeTIQ on dopamine metabolism; and neuroprotective properties of TIQ and 1MeTIQ in vitro and in vivo. Finally, antiaddictive properties of 1MeTIQ will be described in cocaine self-administered rats. Findings implicate TIQ and especially its methyl derivative 1MeTIQ in unique and complex mechanisms of neuroprotection in various neurodegenerative illnesses of the central nervous system. We believe that MAO inhibition, free radicals scavenging properties, and antagonism to the glutamatergic system may play an essential role in neuroprotection. In addition, the results strongly support the view that 1MeTIQ has a considerable potential as a drug for combating substance abuse, through the attenuation of craving.
\end{abstract}

Keywords TIQ - 1MeTIQ - Brain dopamine metabolism . Neuroprotection · Addiction $\cdot$ Rat

L. Antkiewicz-Michaluk ( $₫)$. A. Wąsik · J. Michaluk Department of Neurochemistry, Institute of Pharmacology Polish Academy of Sciences, Smetna Str.12, 31-343 Kraków, Poland e-mail: antkiew@if-pan.krakow.pl

\section{Introduction}

1,2,3,4-Tetrahydroisoquinoline (TIQ) is a member of a family of tetrahydroisoquinolines widespread in plant and animal and human brains (McNaught et al. 1998; Rommelspacher and Susilo 1985). In most cases, tetrahydroisoquinolines can be formed as condensation products of biogenic amines (i.e., phenylethylamines and catecholamines) with aldehydes or $\alpha$-keto acids by the so-called Pictet-Spengler reaction (Rommelspacher and Susilo 1985; Zarranz de Ysern and Ordonez 1981; Nagatsu 1997; McNaught et al. 1998), although some of them may be also synthesized enzymatically (Yamakawa and Ohta 1997, 1999; Naoi et al. 2004). The tetrahydroisoquinoline family can be divided into compounds with catechol- and non-catechol structure. TIQ is the simplest representative of the group of non-catechol tetrahydroisoquinolines which occur naturally in plants and in a variety of food products (Makino et al. 1988; Niwa et al. 1989) as well as in the brain of humans, primates, and rodents (Kohno et al. 1986; Makino et al. 1988; Niwa et al. 1987; Ohta et al. 1987; Yamakawa et al. 1999).

For the first time, tetrahydroisoquinolines attracted considerable attention of neurochemists and pharmacologists when Davis and Walsh (1970) demonstrated that the alcohol metabolite acetaldehyde promoted in vitro conversion of $\left[{ }^{14} \mathrm{C}\right]$ dopamine into $\left[{ }^{14} \mathrm{C}\right]$ tetrahydropapaveroline (THP). Simultaneously, THP was identified in the urine of parkinsonian patients on L-DOPA (3,4-dihydroxyphenylalanine) medication (Sourkes 1971; Sandler et al. 1973; Matsubara et al. 1992) and in the urine and brain of rats treated with LDOPA (Turner et al. 1974). Almost at the same time, salsolinol (6,7-dihydroxy-1-methyl-1,2,3,4-TIQ), an adduct of dopamine and acetaldehyde, was identified in the urine of non-pathologic human volunteers, occurring at high 
concentrations in the urine of intoxicated alcoholics (Collins et al. 1979) and in brains of rats treated with ethanol (Collins and Bigdeli 1975). Although TIQ has been proposed to be one of the etiological factors of Parkinson's disease (PD), its implication in the pathogenesis is not clear, in contrast to other tetrahydroisoquinolines with rather neurotoxic mechanism of action in the brain, e.g., salsolinol or 1-benzyl1,2,3,4-TIQ (1BnTIQ).

Early studies on tetrahydroisoquinolines revealed their neuroleptic-like properties (Ginos and Doroski 1979) and our more recent results suggest that TIQ and its derivatives are antagonists of agonistic conformation of the dopamine $\mathrm{D}_{2}$ receptor (Antkiewicz-Michaluk et al. 2007; Vetulani et al. 2001, 2003a). This explains why TIQ and its congeners effectively block dopaminergic stimulation without affecting much the basal locomotor activity. Pharmacologically tetrahydroisoquinolines aroused also an interest as potential NMDA ( $N$-methyl-D-aspartate) receptor antagonists (Ortwine et al. 1992). Some of them were described as effective antagonists of the phencyclidine (PCP) site (Rogawski et al. 1989). However, most tetrahydroisoquinolines do not substitute for PCP (Nicholson and Balster 2003).

Apart from TIQ, this group encompasses also, the methyl derivative of TIQ, 1-methyl-1,2,3,4-TIQ (1MeTIQ), is a neuroprotective compound. Among several endogenous TIQs 1MeTIQ has a special position, as very early it was described in the brain (Kohno et al. 1986; Makino et al. 1990; Niwa et al. 1987; Ohta et al. 1987), and shortly thereafter recognized as a potential antiparkinsonian agent on the basis of reversal of bradykinesia induced by 1-methyl-4-phenyl-1,2,3,6-tetrahydropyridine (MPTP), TIQ, or 1BnTIQ (Tasaki et al. 1991; Kotake et al. 1995). 1-MeTIQ was identified in normal rat brains in 1986 (Kohno et al. 1986), and subsequently found to be present in foods rich in 2-phenylethylamine, from which it may enter the brain (Makino et al. 1988). However, it is also synthetized enzymatically in the brain from 2-phenethylamine to pyruvate (Niwa et al. 1990; Tasaki et al. 1993; Yamakawa and Ohta 1997). Having an asymmetric carbon atom, 1MeTIQ may appear in the form of R- and S-stereoisomers, and the product found in brain and in foods is a racemate (Makino et al. 1990), although the stereoisomers do not much differ in their biological actions (Abe et al. 2001; Wąsik et al. 2012). 1MeTIQ unlike several other TIQs displays neuroprotective and antiaddictive properties which will be particularly emphasized in this article (Antkiewicz-Michaluk and Vetulani 2001; AntkiewiczMichaluk et al. 2003, 2004, 2006; Wąsik et al. 2007, 2010).

This article reviews some important aspects concerning the chemistry, distribution, pharmacology, and mechanism of action of TIQ and its methyl derivative, 1MeTIQ the simplest representatives of the unsubstituted non-catechol tetrahydroisoquinolines in the mammalian brain.

\section{Synthesis of 1MeTIQ in the Brain}

1MeTIQ was identified in normal rat brains by Kohno and coworkers (Kohno et al. 1986), and subsequently found to be present in foods rich in 2-phenylethylamine, which readily penetrates into the brain across the blood-brain barrier (Makino et al. 1988). 1MeTIQ may be also synthesized enzymatically in the brain (Niwa et al. 1990). The enzyme involved in that process called 1MeTIQase was localized in the mitochondrial-synaptosomal fraction of rat brain, isolated, and purified (Niwa et al. 1990; Tasaki et al. 1993). Its activity is spread throughout the brain, the highest activity being observed in the dopaminergic areas that are implicated in the etiology of PD [striatum and substantia nigra (SN)] and in the cortex. During aging the activity of 1MeTIQase falls (by approximately 40-50\%) in the areas of its highest activity (Absi et al. 2002). 1 MeTIQase may be important in the pathogenesis of PD. The cerebral concentration of 1MeTIQ in normal rat brains was recently determined as $3.5 \mathrm{ng} / \mathrm{g}$ tissue, exceeding several times (three- to fivefold) the concentrations of other simple tetrahydroisoquinolines (Inoue et al. 2008). Most of the studies on 1MeTIQ were carried out on the brains of rodents, but the results on monkeys demonstrate that the regional distribution of $1 \mathrm{MeTIQ}$, other simple tetrahydroisoquinolines, and 1MeTIQase activity are correlated (Yamakawa et al. 1999).

It was also demonstrated that 1MeTIQ synthesis is inhibited by agents that induce experimental parkinsonism (Igarashi et al. 1999; Tasaki et al. 1991; Yamakawa and Ohta 1999). Interestingly, the 1MeTIQ concentration in SN declines in parkinsonian patients as well as in aged rats, by as much as $50 \%$ (Ayala et al. 1994). All those data indicate that the change in 1MeTIQ content of brain may play an important role in the pathogenesis of the toxin-induced parkinsonism, and that the degeneration of dopaminergic neurons may proceed as a result of the loss of neuroprotection afforded by $1 \mathrm{MeTIQ}$. Thus, it seems that $1 \mathrm{MeTIQ}$ is an endogenous substance that protects mainly dopamine cells against free radical damage.

\section{Neuroprotection}

Free Radical Scavenging Properties Afforded by TIQ and $1 \mathrm{MeTIQ}$

To determine whether TIQ and 1MeTIQ may protect against oxidative stress we investigated their capacity to inhibit hydroxyl radical generation in vitro. Oxidative stress leads to the production of reactive oxygen species (Harman 1981), such as superoxide anion radical and hydroxyl radical $(\mathrm{OH})$-chemical species known to damage cellular 
macromolecules (e.g., lipids, sugars, proteins), and this damage can lead to equally damaging secondary products (Sayre et al. 2008). Based on these data, oxidative stress was long regarded as a universal mechanism of inducing cell death (Dykens 1999). In the brain, the main source of toxic $\mathrm{OH}$ formation and $\mathrm{H}_{2} \mathrm{O}_{2}$ generation is $\mathrm{MAO}$, consequent to monoamine deamination (for review see Singer and Ramsay 1995). Similarly, excessive intraneuronal dopamine catabolism by MAO augments the formation of free radicals in the brain. A direct study on the free radical scavenging capacity of other tetrahydroisoquinolines was carried out in vitro in rodent brain. Both TIQ and 1MeTIQ, in contrast to other tetrahydroisoquinolines (e.g., 1BnTIQ and salsolinol), inhibit MAOA and MAOB activities and possess other antioxidant properties (Patsenka and Antkiewicz-Michaluk 2004), as indicated by the effects of TIQ and 1MeTIQ to inhibit free radical formation and abolish dopamine generation of $\mathrm{OH}$ via the Fenton reaction (Antkiewicz-Michaluk et al. 2006). Those results demonstrate that TIQ and 1MeTIQ, independent of direct interaction with biological structures, possess intrinsic antioxidant properties.

\section{Neuroprotection in Relation to Dopaminergic}

Mechanisms in In Vitro and Ex Vivo Studies

Interaction with presynaptic dopamine receptors was investigated by studying 1MeTIQ-induced displacement of dopamine receptor ligands from their binding sites. In general, tetrahydroisoquinolines do not displace antagonistic ligands bound to dopamine $\mathrm{D}_{2}$ receptors (AntkiewiczMichaluk et al. 2007; Vetulani et al. 2003a), the exception being $\left[{ }^{11} \mathrm{C}\right]$ raclopride. Depression of binding of $\left[{ }^{11} \mathrm{C}\right] \mathrm{ra}-$ clopride may be interpreted as the sign of increased synaptic dopamine concentrations, which competes with raclopride at dopamine $\mathrm{D}_{2}$ receptor sites (Laruelle 2000). Tetrahydroisoquinolines were shown to displace $\left[{ }^{11} \mathrm{C}\right]$ raclopride, and the (S)-enantiomers of TIQ and 1MeTIQ were most potent in this respect. These findings suggest that tetrahydroisoquinoline analogs profoundly stimulate dopamine release, resulting in the competitive inhibition of $\left[{ }^{11} \mathrm{C}\right]$ raclopride binding to dopamine $\mathrm{D}_{2}$ receptors, but not loss of receptor number (Ishiwata et al. 2001). The dopamine receptor agonist $\left[{ }^{3} \mathrm{H}\right]$ apomorphine was another ligand displaced from dopamine $\mathrm{D}_{2}$ receptors by tetrahydroisoquinolines (Antkiewicz-Michaluk et al. 2007; Vetulani et al. 2003a). In contrast to antagonists, an agonist radioligand binds preferentially to the high-affinity state and is expected to have greater sensitivity to dopamine, the endogenous agonist. Thus, the experiments with $\left[{ }^{3} \mathrm{H}\right]$ apomorphine displacement confirm that tetrahydroisoquinolines may release dopamine from dopaminergic terminals. However, owing to their MAO-inhibiting properties tetrahydroisoquinolines do not cause neurodegeneration of dopaminergic neurons.
In biochemical studies, MPTP and pro-parkinsonian $\beta$-carbolines potently inhibited the activity of $1 \mathrm{MeTIQ}$-ase (Yamakawa and Ohta 1999). It is well established by behavioral, biochemical ex vivo but also in vivo microdialysis studies, that both enantiomers (R)- and (S)- as well as racemic (R,S)-1MeTIQ demonstrate neuroprotective activity, as evidenced by their attenuation of the behavioral and biochemical effects of dopaminergic neurodegeneration induced by experimental neurotoxins such as: MPTP, 1BnTIQ, and rotenone (Antkiewicz-Michaluk et al. 2003, 2004, 2011; Kotake et al. 1995, 2005; Tasaki et al. 1991).

Several tetrahydroisoquinolines and their congeners, including TIQ and 1MeTIQ, interfere with MAO activity, indicating putative neuroprotection relating to the pathogenesis of PD (Naoi and Maruyama 1993). Subsequently, Thull et al. (1995) investigated 45 isoquinoline derivatives and found most of them to be reversible inhibitors of MAOA and MAOB, with preferential effects on the A form. Their studies brought to the forefront the question of the physiological significance of endogenous MAO inhibitors, and a suggested role for endogenous tetrahydroisoquinolines in the control of neurotransmitter function, and prevention of neurotoxicity related to MAO activity in the brain.

The data from ex vivo neurochemical experiments have shown stereospecificity of 1MeTIQ enantiomers, (R)- and (S)- in respect of their effects on dopamine catabolism. While both enantiomers increased the concentrations of dopamine and its extraneuronal metabolite, 3-methoxytyramine (3-MT) in rat striatum, they differently affected dopamine catabolism. Thus, (R)-1MeTIQ increased both the level of the final dopamine metabolite homovanillic acid (HVA) (by about $70 \%$ ) and the rate of dopamine metabolism (by $50 \%$ ), while (S)-1MeTIQ depressed the DOPAC (3,4-dihydroxyphenylacetic acid) and HVA levels (by 60 and $40 \%$, respectively), and attenuated the rate of dopamine metabolism (Antkiewicz-Michaluk et al. 2011). These data suggest that the (S)-enantiomer may offer better and more effective protection against neurotoxicity. It would be important to mention that even after chronic administration a high dose of 1MeTIQ never produced noxious effects on dopamine neurons (Antkiewicz-Michaluk et al. 2001).

Showing structural resemblance to MPTP, the potent neurotoxin capable of producing persistent parkinsonism in humans (Langston et al. 1983) and in laboratory animals (Jenner and Marsden 1986), initially all tetrahydroisoquinolines were assumed to be neurotoxic to dopamine neurons. In fact, the early studies reported that they generally are neurodegenerating agents (Suzuki et al. 1990), the most neurotoxic being 1BnTIQ, and $N$-methyl derivatives, (R)1,2-dimethyl-5,6-dihydroxy-TIQ, (R)- $N$-methyl-salsolinol, and TIQ (Nagatsu 1997). This finding contrasted with an earlier report which found no neurotoxicity of tetrahydroisoquinolines on nigrostriatal dopamine neurons (Perry et al. 
1988). The most recent studies, in which the actions of 1MeTIQ and TIQ were directly compared, suggest that TIQ, in fact, produces some damage to dopaminergic neurons, as reflected by a mild but significant decrease in the striatal dopamine concentration in rats chronically administered TIQ in high doses (50-100 mg/kg). In contrast, 1MeTIQ has never been shown to produce a decline in dopamine in brain, although both of these tetrahydroisoquinolines similarly affect dopamine catabolism (Antkiewicz-Michaluk et al. 2000a; 2001; Antkiewicz-Michaluk and Vetulani 2001).

\section{Neuroprotection of 1MeTIQ Against Rotenone}

Rotenone, a natural compound, is a classical, lipophilic inhibitor of mitochondrial complex I (Gutman et al. 1970; Horgan et al. 1968), and selectively toxic to dopaminergic neurons (Marey-Semper et al. 1993). Rotenone, an environmental toxin induces the formation of Lewy bodies, which are the most characteristic histopathological feature of Parkinson's disease (Betarbet et al. 2000), and may be used to produce a parkinsonian syndrome more realistic from $\mathrm{MPP}^{+}$ animal modeling of PD. A defect of mitochondrial function due to complex I inhibition was postulated to be the cause of rotenone-induced neurodegeneration (Jenner 2001; Greenamyre et al. 2001). Rotenone also causes dopamine release, as evidenced by microdialysis and neurochemical data (Santiago et al. 1995; Thiffault et al. 2000), and this may also contribute to the degeneration of dopaminergic neurons. In our studies, rotenone administered in a single dose did not produce evident behavioral or biochemical effect. In contrast, repeated administration of rotenone $(12 \mathrm{mg} / \mathrm{kg}$ s.c.) causing abnormalities in general behavior produced considerable mortality and dramatic increases in dopamine metabolism, which may be ascribed to an increase in the oxidative pathway, and strongly depressed the concentration of the extracellular dopamine metabolite, 3-MT. These behavioral and biochemical changes were effectively counteracted by administration of 1MeTIQ before each dose of rotenone (Antkiewicz-Michaluk et al. 2003). In addition, rotenone administered intracerebrally to the left medial forebrain bundle (MFB) produced neurodegeneration of dopamine neurons in extrapyramidal system (a considerable decrease in dopamine and its metabolite levels) without affecting the serotonin system (Antkiewicz-Michaluk et al. 2004). Those changes were observed 21 days after the intracerebral injection of rotenone, they suggest a durable neurotoxic effect. Peripheral administration of $1 \mathrm{MeTIQ}$ $(50 \mathrm{mg} / \mathrm{kg}$ i.p.) before, and then daily for 21 days, significantly reduced the fall of striatal dopamine concentration (Antkiewicz-Michaluk et al. 2004). The above data suggest that $1 \mathrm{MeTIQ}$ is able to counteract the damaging action of dopaminergic neurotoxin, rotenone and seems to be a potential neuroprotective agent.
Neuroprotection of 1MeTIQ Against GlutamateEvoked Neurotoxicity

Recently, it was demonstrated that 1MeTIQ shares many activities with TIQ, and found that the compounds similarly inhibit free radical generation in an abiotic system, as well as indices of neurotoxicity (caspase- 3 activity and lactate dehydrogenase release) induced by glutamate in mouse embryonic primary cell cultures (Antkiewicz-Michaluk et al. 2006). However, in granular cell cultures obtained from 7-day-old rats, 1MeTIQ (in concentration-related manner) prevented glutamate-induced cell death and ${ }^{45} \mathrm{Ca}^{2+}$ influx, whereas TIQ did not. Such profile of action of 1 MeTIQ suggested specific effects of this compound on an excitatory amino acids receptor. In addition, it was shown in an in vivo microdialysis experiment that $1 \mathrm{MeTIQ}$ prevents kainateinduced release of excitatory amino acids from the rat frontal cortex (Antkiewicz-Michaluk et al. 2006).

Comparing the chemical structure of 1MeTIQ with other known compounds containing TIQ skeleton and their molecular mechanism of action, one can find similarities between 1MeTIQ and these derivatives which are noncompetitive AMPA/kainate receptor antagonists that protect animals in the maximal electroshock seizure, pentylenetetrazole and audiogenic DBA/2 mouse seizure models (Ferreri et al. 2004; Gitto et al. 2003). In fact, 1MeTIQ exerts anticonvulsant effects, increasing the threshold for electroconvulsions and potentiation of the antiseizure action of carbamazepine and valproate against maximal electroshock (Luszczki et al. 2006). In the light of all these experiments, 1 MeTIQ offers a unique and complex mechanism of neuroprotection in which inhibitory effect on MAO connected with free radicals scavenging properties, and antagonism to the glutamatergic system seems to play a very important role.

\section{Addiction}

Addiction is a complex disease process of the brain which results from recurring drug intoxication and is modulated by genetic, experiential, and environmental factors. Drug addiction is one of the most difficult medical and social problems, as no effective pharmacotherapy has been available so far. Until recently, it was believed that addiction was associated with neuroplasticity in the cortico-striatal brain circuitry, which is important for adaptive behavior and predominantly involved reward processes mediated by limbic circuits, whereas results from recent neuroimaging studies have implicated additional brain areas, especially the frontal cortex (Goldstein and Volkov 2002). Drug addiction is often defined by the pharmacological terms: tolerance, sensitization, dependence, and withdrawal. Tolerance refers to the phenomenon where repeated administration of a drug 
at the same dose causes a diminishing effect or a need for an increasing drug dose to produce the same effect. Sensitization refers to the opposite condition where repeated administration of the same drug dose produces an escalating effect. Interestingly, the same drug can simultaneously evoke tolerance and sensitization to its numerous diverse effects (e.g., in the case of morphine, tolerance to its analgesic effect and sensitization to its locomotor effect). Dependence is defined as a need for continual drug exposure to avoid a withdrawal syndrome which is characterized by physical or motivational disturbance when the drug is withdrawn.

The neurobiological changes that accompany drug addiction have not been understood so far; however, drugs of abuse are unique in terms of their reinforcing properties. Dopaminergic mechanisms are a traditional target in the field of addiction, as the acute rewarding effects of addictive drugs are mediated by enhancing dopamine transmission; moreover, dopamine release reinforces reward learning (Berridge and Robinson 1998; Kelley 2004a, b). A question arises about the neurobiological substrate of reward. The nucleus accumbens (NAc) as a ventral striatum is considered to be a crucial point of integration of information by receiving emotional and cognitive inputs, and by projecting to motor output regions (Mogenson et al. 1980; Kelley 2004a). The NAc, along with the hippocampus, frontal cortex (FCx), and basolateral amygdala, receives dopamine input from the ventral tegmental area (VTA); furthermore, as it has been shown by many others, the majority of dopamine neurons that innervate the forebrain are located in the midbrain, specifically in the VTA and SN (Fallon and Loughlin 1995; Pitkanen 2000). The SNc innervates the dorsal striatum (caudate-putamen), whereas the VTA provides an input to the rest of the forebrain, including the ventral striatum (NAc), FCx, amygdala, and hippocampus. Early theories on drugs of abuse and natural rewards suggested that activation of dopamine neurons in VTA, and the release of dopamine in target structures signaled reward, especially in the NAc (Di Chiara 2002; Ungless 2004). However, aversive stimuli also increase dopamine release in a variety of brain structures, which indicates a role of dopamine beyond reward (Inglis and Moghaddam 1999). It is noteworthy that some evidence points to differential dopamine responses to aversive versus rewarding stimuli (Schultz 2002, 2010). Some recent studies have also shown that the glutamate system and its release is an important factor in drug addiction, and that imbalance in glutamate homeostasis engenders changes in neuroplasticity, which impair communication between the prefrontal cortex and the NAc (Kalivas 1995; Ma et al. 2006; Nagy 2004; Popik et al. 1998). In a clinical setting, neuroimaging studies have shown that cue or drug exposure increased the activity of
$\mathrm{FCx}$ and NAc, as well as self-reported drug craving in cocaine addicts (Goldstein and Volkov 2002). In animal models, a challenge of cocaine or heroin increases the synaptic release of glutamate in cocaine- or heroin-withdrawn rats as a result of the activation of corticostriatal pathways; and on the other hand, inactivation of the corticostriatal pathway has been shown to be effective in inhibiting cocaine- or heroin-induced drug seeking behavior (Kalivas et al. 2005).

\section{Cocaine Addiction: The Effect of 1MeTIQ}

Regardless of the mechanism of action of drugs of abuse, the essential role of the mesolimbic dopaminergic system in addiction has been well established (Goldstein and Volkov 2002; Grimm et al. 2003; Moore et al. 1998a, b); to this end, several antidopaminergic drugs were tested as potential anti-abuse agents (Berger et al. 1996; Smelson et al. 2004). Although neuroleptics were previously found not to be useful in this respect, partial agonists of the dopamine $D_{2}$ and $D_{3}$ receptor aroused some hopes (Campiani et al. 2003; Le Foll et al. 2005; Mach et al. 2004). Furthermore, a dopamine reuptake inhibitor could be expected to partially substitute for cocaine and other drugs of abuse, hence self-administration would be diminished and craving minimized (Ritz et al. 1987; Wilcox et al. 2000; Carroll et al. 2004). This type of substitution pharmacotherapy has been found to be highly effective in the treatment of nicotine and heroin addiction (methadone). Hence, studies of partial agonists with an antidopaminergic profile of action different from that of classic neuroleptics seem justified. In the light of the above data, 1MeTIQ is an interesting candidate for future clinical studies.

A vast body of evidence indicates that, apart from the dopaminergic system, also the glutaminergic system is involved in the addiction to drugs of abuse. Hence, of special interest are the observations that 1MeTIQ antagonizes the kainate-induced release of glutamate and aspartate in rat $\mathrm{FCx}$ and shows neuroprotection against the excitotoxicity produced by glutamate in cultured cells (Antkiewicz-Michaluk et al. 2006). In addition, 1 MeTIQ antagonizes the MK-801-produced behavioral and neurochemical effects (Pietraszek et al. 2009) and shares tolerance to excitotoxicity in rat with some well-established uncompetitive NMDA receptor antagonists (Kuszczyk et al. 2010). The latest results reveal a new mechanism of the 1MeTIQ-evoked neuroprotection based on the induction of neuronal tolerance to excitotoxicity.

It is well known that acute administration of the drugs of abuse: cocaine, amphetamine (psychostimulants), and opiates increases the locomotor activity of animals. Repeated administration of the drug of abuse induces 
neurobiological changes, such that after 10 days of withdrawal, acute administration of the drug produces an even greater increase in locomotor activity, a process called sensitization. Both behavioral sensitization, self-administration, and drug-reinstatement of seeking behavior are the major models of drug addiction (Pierce and Kalivas 1997). The compounds which antagonize locomotor sensitization and self-administration in animals may demonstrate antiaddictive properties in a clinic (Narayanan et al. 1996).

Exogenously applied 1MeTIQ significantly antagonized cocaine-induced locomotor sensitization, cocaine selfadministration, and cocaine-induced reinstatement of seeking behavior (Filip et al. 2007; Wạsik et al. 2010). The phenomenon is of interest, as it might be caused by the same mechanisms as those responsible for psychoses or craving for drugs of abuse in humans abusing cocaine or other psychostimulants (Robinson and Berridge 1993; Segal et al. 1981). Both clinical and preclinical studies indicate that the behavioral response to cocaine, including the discriminative stimulus and rewarding effects as well as reinstatement of cocaine seeking behavior, depends on the drug's ability to block the dopamine transporter (Di Chiara 1995; Heidbreder and Hagan 2005). As 1MeTIQ produced parallel decreases in cocaine self-administration and cocaine-induced relapse, the compound may suppress the motivation for drug seeking by decreasing the reinforcing effects of cocaine, and generally by attenuation of the reinforcing effect of drugs of abuse. In fact, activation of both the dopaminergic and the glutaminergic systems has significance in altering the maintenance of cocaine self-administration (Cornish et al. 1999; Pulvirenti et al. 1992), and drug-priming-induced reinstatement of cocaine seeking (Ito et al. 2002; Kalivas and McFarland 2003).

1MeTIQ's inhibitory mechanism on cocaine maintained responding and relapse may include complex interactions with both dopaminergic and/or glutaminergic transmission.

\section{Neurochemical Changes Produced by 1 MeTIQ in Cocaine-Dependent Rats}

Cocaine, a potent inhibitor of monoamine transporters, belongs to the most powerful addictive substances in humans and its abuse has a high risk of relapse (Gawin 1991). Studies on the involvement of biogenic amines in cocaine addiction have shown a contribution of dopamine and serotonin to the maintenance of cocaine self-administration, extinction, and reinstatement of drug-seeking behavior. Dopamine was the amine investigated the most extensively, possibly because of its assumed role as the main neurotransmitter of reward (Di Chiara et al. 2004). It was demonstrated by several authors during cocaine self-administration, that extracellular concentrations of dopamine and serotonin in the striatum increase (Di Ciano et al. 1995; Gratton and Wise 1994;
Wassik et al. 2010), but the rate of monoamines metabolism and the concentrations their metabolites in brain structures decrease (Antkiewicz-Michaluk et al. 2006). The functional significance of the observed depression of the level of monoamine metabolites requires explanation. The determination of changes in metabolism rate yields information about efficiency of the neurotransmitter system. Depending on the state of receptor and the rate of synthesis of a neurotransmitter, the changes in metabolite levels in the same direction may have different consequences. Activation of a receptor (e.g., dopamine receptor) during stabilized selfadministration of cocaine would result in a feedback inhibition of neurotransmitter release by activation of autoreceptors, and the depression of neurotransmitter metabolites without significant changes in neurotransmitter concentration in the neuron. This is reflected by a decrease in the neurotransmitter metabolism index. As the stimulation is indirect, through inhibition of neurotransmitter reuptake, the interaction between the neurotransmitter and receptor is enhanced rather than depressed. On the contrary, during cocaine withdrawal, dopamine and noradrenaline concentrations as well as concentration of their metabolites were diminished, suggesting cocaine-induced impairment in the function of catecholamine neurons, and a consequent decrease in the synthesis and release of neurotransmitters (Parsons et al. 1995; Weiss et al. 1992; Antkiewicz-Michaluk et al. 2006).

While catecholamines seem to be involved in cocaine addiction, the role of serotonin seems to be limited. Specific serotonin agonists do not seem to have significant reinforcing efficacy (Locke et al. 1996). Clinical findings also indicate inefficiency of serotonergic manipulation in combating cocaine dependence (Lima et al. 2003). Although, serotonin metabolism is inhibited in the presence of cocaine, in contrast to alterations in catecholaminergic system, the change was transient.

Basically, a chronic cocaine self-administration similarly to passive administration suppresses the metabolism — both synthesis and release of monoamines in several brain structures (Karoum et al. 1990; Trulson and Ulissey 1987; Antkiewicz-Michaluk et al. 2006). The changes in catecholamine metabolism persist for a considerable period after cessation of cocaine self-administration, suggesting a long-lasting functional impairment of dopamine and noradrenaline systems. In contrast, changes in the serotonergic system are transient, showing the lack of involvement of serotonin in long-term consequences of cocaine exposure (Antkiewicz-Michaluk et al. 2006).

It is suggested that 1MeTIQ is a potential anti-abuse agent, as the drugs which reduce cocaine seeking behavior also reduce cocaine craving (Fuchs et al. 1998; Baker et al. 2001). The possible anti-abuse properties of 1MeTIQ are particularly interesting, as the compounds of this group are proposed to act 
as regulators of brain homeostasis (Antkiewicz-Michaluk et al. 2000a, b; Vetulani 2001). The question arises whether $1 \mathrm{MeTIQ}$ can reach the brain in concentrations producing pharmacological effects? In contrast to catechol TIQs (e.g., salsolinol), non-catechol TIQs such as TIQ and 1MeTIQ penetrate to the brain easily and induce a variety of central effects. No direct measurements of 1MeTIQ concentration in the brain after peripheral administration of the compound have been carried out, but its close congener, TIQ after administration of $40 \mathrm{mg} / \mathrm{kg}$ reached the concentration of $250 \mathrm{nmole} / \mathrm{g}$ in rat (Lorenc-Koci et al. 2004).

In cocaine-dependent rats receiving a priming dose of cocaine in the presence of previously administered 1MeTIQ, the concentration of dopamine in limbic structures was significantly higher than in rats receiving cocaine alone. It might be assumed that the blockade of reinstatement by 1 MeTIQ is related to this effect (Antkiewicz-Michaluk et al. 2007). There is the long established view that depression of dopaminergic activity in the limbic structures may be responsible for craving (Rossetti et al. 1992; Little et al. 1996). Because 1MeTIQ elevates the concentration of dopamine preferentially in limbic structures (NAc) in cocaine-dependent rats, and at the same time inhibits dopamine metabolism in structures containing cell bodies (SN, VTA), 1MeTIQ may be responsible for its inhibition of reinstatement (Antkiewicz-Michaluk et al. 2007).

Another neurochemical action of 1MeTIQ, possibly related to its anticraving effect, is activation of the noradrenergic system in the brain. This effect may be related to the antagonistic action of 1MeTIQ on alpha-2-adrenoceptors. Such an activity was described previously for other, closely related TIQs (Michaluk et al. 2002; Vetulani et al. 2003a, b). The ability of 1MeTIQ to increase the level of the main metabolite of noradrenaline in CNS, 3-methoxy4-hydroxyphenylglycol (MHPG), as well as its extraneuronal metabolite normetanephrine (NM), reflects the antagonistic effect of 1MeTIQ on the alpha-2-adrenoceptor (Antkiewicz-Michaluk, unpublished data). Inhibition of alpha-2-adrenoceptors would result in an increase in noradrenaline release from the nerve endings, and consequently activation of the noradrenergic system.

In the light of the recent experimental data it looks as though serotonin plays a less important role in the phenomenon of cocaine reinstatement. It was shown that cocaine depresses serotonin metabolism only transiently, and that the changes do not persist throughout the withdrawal period, in contrast to dopamine and noradrenaline systems (Antkiewicz-Michaluk et al. 2007).

\section{Morphine Addiction and the Effect of 1MeTIQ}

Morphine acts through activation of opioid $\mu$-receptors and produces the antinociceptive effect called analgesia. It is well known that activation of opioid $\mu$-receptors is closely related with inhibition of calcium uptake and this process is responsible for opioid-induced analgesia (Kamikubo et al. 1983; Chapman and Way 1982). 1MeTIQ administered alone was shown to have a slight antinociceptive effect in the "hot-plate" test in rats, but co-administration of morphine strongly potentiated its analgesia (Wạsik et al. 2007; Vetulani et al. 2003a, b). Moreover, 1MeTIQ applied before each morphine injection completely inhibited the development of morphine tolerance, and prevented naloxone-induced precipitation of the abstinence syndrome (head-twiches and body weight loss) in morphine-dependent rats (Wąsik et al. 2007). A question arises as to the mechanism responsible for 1MeTIQ-induced potentiation of morphine-analgesia, prevention of morphine-produced tolerance, and abstinence syndrome. Some have postulated that the activity of MAO, the enzyme crucial for monoamine and special dopamine catabolism, and the production of free radicals, play an important role in opiate analgesia, tolerance, and dependence (Garzon et al. 1979; Grassing and He 2005). In fact, the irreversible MAOB inhibitor deprenyl and other antioxidants such as vitamin $\mathrm{C}$ produced an increase in morphine antinociception and prevention of the development of morphine tolerance and physical dependence in rodents (Sánchez-Blázquez et al. 2000; Khanna and Sharma 1983). 1MeTIQ, a neuroprotective substance, inhibits MAO and possesses free radical scavenging properties (Antkiewicz-Michaluk et al. 2006). This mechanism would be partially responsible for its antinociception and antiaddictive effects. In addition, it has been shown that 1MeTIQ is also effective in prevention of morphine-induced place preference and alcohol intake (Antkiewicz-Michaluk et al. 2005).

Moreover, others have shown that morphine did not effectively trigger the processes leading to development of tolerance and dependence when administered during $\mathrm{Ca}^{2+}$ channel blockade. Blockade of the voltage-dependent L-type $\mathrm{Ca}^{2+}$ channels effectively facilitates the analgesic action of morphine and prevents the behavioral and neurochemical signs of naloxone-precipitated abstinence syndrome (Contreras et al. 1988; Del Pozo et al. 1987; Michaluk et al. 1998). NMDA glutamate receptor activation and associated $\mathrm{Ca}^{2+}$ influx may also be involved in the induction of morphine sensitization (Vanderschuren and Kalivas 2000). It should be taken into account that 1MeTIQ prevented glutamate-induced cell death and ${ }^{45} \mathrm{Ca}^{2+}$ influx in granular cell cultures (AntkiewiczMichaluk et al. 2006). Thus, 1MeTIQ besides the inhibitory influence on the activity of MAO and free radical scavenging properties possesses mild activity at NMDA receptors.

\section{Conclusions}

The experimental data assembled in this review allow for a more precise characterization of the activity of the 
exogenous 1MeTIQ in the mammalian brain, especially in the nigrostriatal dopaminergic system. On the basis of these studies, the following conclusions may be drawn:

- exogenous 1 MeTIQ has high affinity for brain tissue

- 1 MeTIQ is a partial dopamine agonist

- 1MeTIQ reversibly inhibits MAOA and MAOB: in vitro and in vivo studies

- 1MeTIQ is a scavenger of free radicals

- $1 \mathrm{MeTIQ}$ expresses neuroprotective properties

- 1 MeTIQ demonstrates antiaddictive potency

This ability of 1MeTIQ may be of clinical importance and raises hope for its application in neurodegenerative diseases (e.g., Parkinson's disease) and addiction evoked by drugs of abuse.

Acknowledgments Thanks are due to Dr. Jan Boksa (Department of Medicinal Chemistry, Institute of Pharmacology Polish Academy of Sciences) for synthesis of 1MeTIQ. The authors thank the Polish Committee of Scientific Research (project N N401 004836) and the statutory funds of the Institute of Pharmacology Polish Academy of Sciences, Krakow, Poland for financial support.

Open Access This article is distributed under the terms of the Creative Commons Attribution License which permits any use, distribution, and reproduction in any medium, provided the original author(s) and the source are credited.

\section{References}

Abe K, Taguchi K, Wasai T, Ren J, Utsunomiya I, Shinohara T, Miyatake T, Santo T (2001) Stereoselective effect of (R)- and (S)-1-methyl-1,2,3,4-tetrahydroisoquinolines on a mouse model of Parkinson's disease. Brain Res Bull 56:55-60

Absi E, Parrado J, Ayala A, Machado A (2002) Decrease of 1-methyl1,2,3,4-tetrahydroisoquinoline synthesizing enzyme activity in the brain areas of aged rat. Brain Res 955:161-163

Antkiewicz-Michaluk L, Vetulani J (2001) Tetrahydroisoquinolines as endogenous neurotoxins and neuroprotectants. Acta Neurobiol Exp 61:246

Antkiewicz-Michaluk L, Michaluk J, Romanska I, Papla I, Vetulani J (2000a) Antidopaminergic effects of 1,2,3,4-tetrahydroisoquinoline and salsolinol. J Neural Transm 107:1009-1019

Antkiewicz-Michaluk L, Romanska I, Papla I, Michaluk J et al (2000b) Neurochemical changes induced by acute and chronic administration of 1,2,3,4-tetrahydroisoquinoline and salsolinol in dopaminergic structures of rat brain. Neuroscience 96:59-64

Antkiewicz-Michaluk L, Michaluk J, Mokrosz M, Romanska I, Lorenc-Koci E, Ohta S, Vetulani J (2001) Different action on dopamine catabolic pathways of two endogenous 1,2,3,4tetrahydroisoquinolines with similar antidopaminergic properties. J Neurochem 78:100-108

Antkiewicz-Michaluk L, Karolewicz B, Romanska I, Michaluk J, Bojarski A, Vetulani J (2003) 1-Methyl-1,2,3,4-tetrahydroisoquinoline protects against rotenone-induced mortality and biochemical changes in rat brain. Eur J Pharmacol 466:263-269

Antkiewicz-Michaluk L, Wardas J, Michaluk J, Romanska I, Bojarski A, Vetulani J (2004) Protective effect of 1-methyl-1,2,3,4tetrahydroisoquinoline against dopaminergic neurodegeneration in the extrapyramidal structures produced by intracerebral injection of rotenone. Int J Neuropsychopharmacol 7:155-163

Antkiewicz-Michaluk L, Filip M, Kostowski W, Patsenka A et al (2005) 1-Methyl-1,2,3,4-tetrahydroisoquinoline attenuates ethanol, cocaine and morphine addiction in behavioral models: neurochemical correlates. Acta Neurobiol Exp 65:301-321

Antkiewicz-Michaluk L, Lazarewicz JW, Patsenka A, Kajta M, Zieminska E, Salinska E, Wasik A, Golembiowska K, Vetulani J (2006) The mechanism of 1,2,3,4-tetrahydroisoquinolines neuroprotection: the importance of free radicals scavenging properties and inhibition of glutamate-induced excitotoxicity. J Neurochem 97:846-856

Antkiewicz-Michaluk L, Filip M, Michaluk J, Romanska I et al (2007) An endogenous neuroprotectant substance, 1-methyl1,2,3,4-tetrahydroisoquinoline (1MeTIQ), prevents the behavioral and neurochemical effects of cocaine reinstatement in drugdependent rats. J Neural Transm 114:307-317

Antkiewicz-Michaluk L, Wasik A, Romanska I, Bojarski A, Michaluk J (2011) Both stereoselective (R)- and (S)-1-methyl-1,2,3,4tetrahydroisoquinoline enantiomers protect striatal terminals against rotenone-induced suppression of dopamine release. Neurotox Res 20:134-149

Ayala A, Parrado J, Cano J, Machado A (1994) Reduction of 1-methyl 1,2,3,4-tetrahydroisoquinoline level in substantia nigra of the aged rat. Brain Res 638:334-336

Baker DA, Nguyen LTL, Fuchs RA, Neisewander JL (2001) Influence of individual differences and chronic fluoxetine treatment on cocaine-seeking behavior in rats. Psychopharmacology 155:18-26

Berger SP, Hall S, Mickalian JD, Reid MS et al (1996) Haloperidol antagonism of cue-elicited cocaine craving. Lancet 347:504-508

Berridge K, Robinson T (1998) What is the role dopamine in reward: hedonic impact, reward learning, or incentive salience? Brain Res Rev 28:309-369

Betarbet R, Sherer TB, MacKenzie G, Garcia-Osuna M, Panov AV, Greenamyre JT (2000) Chronic systemic pesticide exposure reproduces features of Parkinson's disease. Nat Neurosci 3:1301-1306

Campiani G, Butini S, Trotta F, Fattorusso C et al (2003) Synthesis and pharmacological evaluation of potent and highly selective D3 receptor ligands: inhibition of cocaine-seeking behavior and the role of dopamine D3/D2 receptors. J Med Chem 46:3822-3839

Carroll FI, Runyon SP, Abraham P et al (2004) Monoamine transporter binding, locomotor activity, and drug discrimination properties of 3-(4-substituted-phenyl)tropane-2-carboxylic acid methyl ester isomers. J Med Chem 47:6401-6409

Chapman D, Way E (1982) Modification of endorphin/enkephalin analgesia by divalent cations, a cation chelator and ionophore. $\mathrm{Br}$ J Pharmacol 75:389-396

Collins MA, Bigdeli MG (1975) Tetrahydroisoquinolines in vivo. I. Rat brain formation of salsolinol, a condensation product of dopamine and acetaldehyde, under certain conditions during ethanol intoxication. Life Sci 16:585-601

Collins MA, Nijm WP, Borge GF, Teas G et al (1979) Dopaminerelated tetrahydroisoquinolines: significant urinary excretion by alcoholics after alcohol consumption. Science 206:1184-1186

Contreras E, Tamayo L, Amigo M (1988) Calcium channel antagonists increase morphine-induced analgesia and antagonize morphine tolerance. Eur J Pharmacol 148:463-466

Cornish JL, Duffy P, Kalivas PW (1999) A role for nucleus accumbens glutamate transmission in the relapse to cocaineseeking behavior. Neuroscience 93:1359-1367

Davis VE, Walsh MJ (1970) Alcohol, amines, and alkaloids: a possible biochemical basis for alcohol addiction. Science 167:1005-1007 
Del Pozo E, Caro G, Baeyens EM (1987) Analgesic effects of several calcium channel blockers in mice. Eur J Pharmacol 137:155-160

Di Chiara G (1995) The role of dopamine in drug abuse viewed from the perspective of its role in motivation. Drug Alcohol Depend 38:95-137

Di Chiara G (2002) Nucleus accumbens shell and core dopamine: differential role in behavior and addiction. Behav Brain Res 137:75-114

Di Chiara G, Bassareo V, Fenu S, De Luca MA et al (2004) Dopamine and drug addiction: the nucleus accumbens shell connection. Neuropharmacology 47:227-241

Di Ciano P, Coury A, Depoortere RY, Egilmez Y et al (1995) Comparison of changes in extracellular dopamine concentrations in the nucleus accumbens during intravenous self-administration of cocaine or d-amphetamine. Behav Pharmacol 6:311-322

Dykens JA (1999) Free radicals and mitochondria dysfunction in excitotoxicity and neurodegenerative disease. In: Koliatsos VE, Ratan RR (eds) Death and diseases of the nervous system. Humana Press, Totowa

Fallon JH, Loughlin SE (1995) Substantia nigra. In: Paxinos G (ed) The rat nervous system. Academic Press, San Diego

Ferreri G, Chimirri A, Russo E, Gitto R, Gareri P, De Sarro A, De Sarro G (2004) Comparative anticonvulsant activity of $N$-acetyl1-aryl-6-7-dimethoxy-1,2,3,4-tetrahydroisoquinoline derivatives in rodents. Pharmacol Biochem Behav 77:85-94

Filip M, Antkiewicz-Michaluk L, Zaniewska M, Frankowska M, Golda A, Vetulani J, Przegalinski E (2007) Effects of 1-methyl1,2,3,4-tetrahydroisoquinoline on the behavioral effects of cocaine in rats. J Physiol Pharamacol 58:625-639

Fuchs RA, Tran-Nguyen LTL, Specio SE, Groff RS et al (1998) Predictive validity of the extinction/reinstatement model of drug craving. Psychopharmacology 135:151-160

Garzon J, Fuentes J, Del Rio J (1979) Effect of selective monoamine oxidase inhibitor drugs on morphine tolerance and physical dependence in mice. Neuropharmacology 18:531-536

Gawin FH (1991) Cocaine addiction: psychology and neurophysiology. Science 251:1580-1586

Ginos JZ, Doroski D (1979) Dopaminergic antagonists: effects of 1,2,3,4-tetrahydroisoquinoline and its $N$-methyl and $N$-propyl homologs on apomorphine- and L-dopa-induced behavioral effects in rodents. J Pharmacol Exp Ther 209:79-86

Gitto R, Barreca ML, De Luca L, De Sarro G, Ferreri G, Quartarone S, Russo E, Constanti A, Chimirri A (2003) Discovery of a novel and highly potent noncompetitive AMPA receptor antagonist. J Med Chem 46:197-200

Goldstein RZ, Volkov ND (2002) Drug addiction and its underlying neurobiological basis: neuroimaging evidence for the involvement of the frontal cortex. Am J Psychiatry 159:1642-1652

Grassing K, He S (2005) Effects of high-dose of selegiline on morphine reinforcement and precipitated withdrawal in dependent rats. Behav Pharmacol 16:1-13

Gratton A, Wise RA (1994) Drug- and behavior-associated changes in dopamine-related electrochemical signals during intravenous cocaine self-administration in rats. J Neurosci 14:4130-4146

Greenamyre JT, Betarbet R, Sherer T, Panov A (2001) Parkinson's disease, pesticides and mitochondrial dysfunction. Trends Neurosci 24:247

Grimm JW, Lu L, Hayashi T, Hope BT et al (2003) Time-dependent increases in brain-derived neurotrophic factor protein levels within the mesolimbic dopamine system after withdrawal from cocaine: implications for incubation of cocaine craving. J Neurosci 23:742-747

Gutman M, Singer TP, Beinert H, Casida JE (1970) Reaction sites of rotenone, piericidin $\mathrm{A}$, and amytal in relation to the nonheme iron components of NADH dehydrogenase. Proc Natl Acad Sci USA 65:763-770
Harman D (1981) The aging process. Proc Natl Acad Sci USA 78:7124-7128

Heidbreder CA, Hagan JJ (2005) Novel pharmacotherapeutic approaches for the treatment of drug addiction and craving. Curr Opin Pharmacol 51:107-118

Horgan DJ, Singer TP, Casida JE (1968) Studies on the respiratory chain-linked reduced nicotinamide adenine dinucleotide dehydrogenase. 13. Binding sites of rotenone, piericidin $\mathrm{A}$, and amytal in the respiratory chain. J Biol Chem 243:834-843

Igarashi K, Sugiyama Y, Kasuya F, Saiki K, Yamakawa T, Ohta S (1999) Determination of 1-methyl-1,2,3,4-tetrahydroisoquinoline in mouse brain after treatment with haloperidol by gas chromatography-selected ion monitoring. J Chromatogr B Biomed Sci Appl 731:53-58

Inglis FM, Moghaddam B (1999) Dopaminergic innervation of the amygdala is highly responsive to stress. J Neurochem 72:1088-1094

Inoue H, Matsubara D, Tsuruta Y (2008) Simultaneous analysis of 1,2,3,4-tetrahydroisoquinolines by high-performance liquid chromatography using 4-(5,6-dimethoxy-2-phthalimidinyl)-2methoxyphenylsulfonyl chloride as a fluorescent labeling reagent. J Chromatogr B Anal Technol Biomed Life Sci $867: 32-36$

Ishiwata K, Koyanagi Y, Saitoh T, Taguchi K, Toda J, Sano T, Senda M (2001) Effects of single and repeated administration of 1,2,3,4-tetrahydroisoquinoline analogs on the binding of (11C)raclopride to dopamine D2 receptors in the mouse brain. J Neural Transm 108:1111-1125

Ito R, Dalley JW, Robbins TW, Everitt BJ (2002) Dopamine release in the dorsal striatum during cocaine-seeking behavior under the control of a drug-associated cue. J Neurosci 22:6247-6253

Jenner P (2001) Parkinson's disease, pesticides and mitochondrial dysfunction. Trends Neurosci 24:245-247

Jenner P, Marsden CD (1986) The action of 1-methyl-4-phenyl1,2,3,4-tetrahydropyridine in animals as a model of Parkinson's disease. J Neural Transm Suppl 20:11-39

Kalivas PW (1995) Interaction between dopamine and excitatory amino acids in behavioral sensitization to psychostimulants. Drug Alcohol Depend 37:95-100

Kalivas PW, McFarland K (2003) Brain circuitry and the reinstatement of cocaine-seeking behavior. Psychopharmacology 168:44-56

Kalivas PW, Volkow N, Seamans J (2005) Unmanageable motivation in addiction: a pathology in prefrontal-accumbens glutamate transmission. Neuron 45:647-650

Kamikubo K, Niwa M, Fugimura H, Miura K (1983) Morphine inhibits depolarization-dependent calcium uptake by synaptosomes. Eur J Pharmacol 95:149-150

Karoum F, Suddath RL, Wyatt RJ (1990) Chronic cocaine and rat brain catecholamines: long-term redaction in hypothalamic and frontal cortex dopamine metabolism. Eur J Pharmacol 186:1-8

Kelley AE (2004a) Memory and addiction: shared neuronal circuitry and molecular mechanisms. Neuron 44:161-179

Kelley AE (2004b) Ventral striatal control of appetitive motivation: role in ingestive behavior and reward-related learning. Neurosci Biobehav Rev 27:765-776

Khanna NC, Sharma SK (1983) Megadoses of vitamin C prevent the development of tolerance and physical dependence on morphine in mice. Life Sci 33:401-404

Kohno M, Ohta S, Hirobe M (1986) Tetrahydroisoquinoline and 1-methyltetrahydroisoquinoline as novel endogenous amines in rat brain. Biochem Biophys Res Commun 140:448-454

Kotake Y, Tasaki Y, Makino Y, Ohta S, Hirobe M (1995) 1-Benzyl1,2,3,4-tetrahydroisoquinoline as a parkinsonism-inducing agent: a novel endogenous amine in mouse brain and parkinsonian CSF. J Neurochem 65:2633-2638 
Kotake Y, Taguchi R, Okuda K, Sekiya Y, Tasaki Y, Hirobe M, Ohta S (2005) Neuroprotective effect of 1-methyl-1,2,3,4-tetrahydroisoquinoline on cultured rat mesencephalic neurons in the presence or absence of various neurotoxins. Brain Res 1033:143-150

Kuszczyk M, Slomka M, Antkiewicz-Michaluk L, Salinska E et al (2010) 1-Methyl-1,2,3,4-tetrahydroisoquinoline and established noncompetitive NMDA receptor antagonists induce tolerance to excitotoxicity. Pharmacol Rep 62:1041-1050

Langston JW, Ballard P, Tetrud JW, Irwin I (1983) Chronic parkinsonism in humans due to a product of meperidine-analog synthesis. Science 219:979-980

Laruelle M (2000) Imaging synaptic neurotransmission with in vivo binding competition techniques: a critical review. J Cereb Blood Flow Metab 20:423-451

Le Foll B, Goldberg SR, Sokoloff P (2005) The dopamine D3 receptor and drug dependence: effects on reward or beyond? Neuropharmacology 49:525-541

Lima MS, Reisser AA, Soares BG, Farrell M (2003) Antidepressants for cocaine dependence. Cochrane Database of Systematic Reviews 2 CD002950. doi:10.1002/14651858

Little KY, Patel UN, Clark TB, Butts JD (1996) Alteration of brain dopamine and serotonin levels in cocaine users: a preliminary report. Am J Psychiatry 153:1216-1218

Locke KW, Levesque TR, Nicholson KL, Balster RL (1996) Dexfenfluramine lacks amphetamine-like abuse potential. Prog Neuropsychopharmacol Biol Psychiatry 20:1019-1035

Lorenc-Koci E, Wojcikowski J, Kot M, Haduch A et al (2004) Disposition of 1,2,3,4-tetrahydroisoquinoline in the brain of male Wistar and Dark Agouti rats. Brain Res 996:168-179

Luszczki JJ, Antkiewicz-Michaluk L, Czuczwar SJ (2006) 1-Methyl1,2,3,4-tetrahydroisoquinoline enhances the anticonvulsant action of carbamazepine and valproate in the mouse maximal electroshock seizure model. Neuropharmacology 50:133-142

Ma YY, Guo CY, Yu P, Lee DY et al (2006) The role of NR2B containing NMDA receptor in place preference conditioned with morphine and natural reinforcers in rats. Exp Neurol 200: 343-355

Mach UR, Hackling AE, Perachon S, Ferry S et al (2004) Development of novel 1,2,3,4-tetrahydroisoquinoline derivatives and closely related compounds as potent and selective dopamine D3 receptor ligands. Chem Biochem 5:508-518

Makino Y, Ohta S, Tachikawa O, Hirobe M (1988) Presence of tetrahydroisoquinoline and 1-methyl-tetrahydroisoquinoline in foods: compounds related to Parkinson's disease. Life Sci 43:373-378

Makino Y, Tasaki Y, Ohta S, Hirobe M (1990) Confirmation of the enantiomers of 1-methyl-1,2,3,4-tetrahydroisoquinoline in the mouse brain and foods applying gas chromatography/mass spectrometry with negative ion chemical ionization. Biomed Environ Mass Spectrom 19:415-419

Marey-Semper I, Gelman M, Levi-Strauss M (1993) The high sensitivity to rotenone of striatal dopamine uptake suggests the existence of a constitutive metabolic deficiency in dopaminergic neurons from the substantia nigra. Eur J Neurosci 5:1029-1034

Matsubara K, Fukushima S, Akane A, Kobayashi S et al (1992) Increased urinary morphine, codeine and tetrahydropapaveroline in parkinsonian patient undergoing L-3,4-dihydroxyphenylalanine therapy: a possible biosynthetic pathway of morphine from L-3,4-dihydroxyphenylalanine in humans. J Pharmacol Exp Ther 260:974-988

McNaught KS, Carrupt PA, Altomare C, Cellamare S et al (1998) Isoquinoline derivatives as endogenous neurotoxins in the aetiology of Parkinson's disease. Biochem Pharmacol 56:921-933

Michaluk J, Karolewicz B, Antkiewicz-Michaluk L, Vetulani J (1998) Effects of various $\mathrm{Ca}^{2+}$ channel antagonists on morphine analgesia, tolerance and dependence, and on blood pressure in the rat. Eur J Pharmacol 352:189-197

Michaluk J, Krygowska-Wajs A, Karolewicz B, Antkiewicz-Michaluk L (2002) Role of noradrenergic system in the mechanism of action of endogenous neurotoxin 1,2,3,4-tetrahydroisoquinoline: biochemical and functional studies. Pol J Pharmacol 54:19-25

Mogenson GJ, Jones DL, Yim CY (1980) From motivation to action: functional interface between the limbic system and the motor system. Prog Neurobiol 14:69-97

Moore RJ, Vinsant SL, Nader MA, Porrino LJ et al (1998a) Effect of cocaine self-administration on striatal dopamine D1 receptors in rhesus monkeys. Synapse 28:1-9

Moore RJ, Vinsant SL, Nader MA, Porrino LJ et al (1998b) Effect of cocaine self-administration on striatal dopamine D2 receptors in rhesus monkeys. Synapse 30:88-96

Nagatsu T (1997) Isoquinoline neurotoxins in the brain and Parkinson's disease. Neurosci Res 29:99-111

Nagy J (2004) The NR2B subtype of NMDA receptor: a potential target for the treatment of alcohol dependence. Curr Drug Targets CNS Neurol Disord 3:169-179

Naoi M, Maruyama W (1993) Type B monoamine oxidase and neurotoxins. Eur Neurol 33:31-37

Naoi M, Maruyama W, Nagy GM (2004) Dopamine-derived salsolinol derivatives as endogenous monoamine oxidase inhibitors: occurrence, metabolism and function in human brains. Neurotoxicology 25:193-204

Narayanan S, Willins D, Dalia A, Wallace L, Uretsky N (1996) Role of dopaminergic mechanisms in the stimulatory effects of MK801 injected into the ventral tegmental area and the nucleus accumbens. Pharmacol Biochem Behav 54:565-573

Nicholson KL, Balster RL (2003) Evaluation of the phencyclidinelike discriminative stimulus effects of novel NMDA channel blockers in rats. Psychopharmacology 170:215-224

Niwa T, Takeda N, Kaneda N, Hashizume Y, Nagatsu T (1987) Presence of tetrahydroisoquinoline and 2-methyl-tetrahydroisoquinoline in parkinsonian and normal human brains. Biochem Biophys Res Commun 144:1084-1089

Niwa T, Yoshizumi H, Tatematsu A, Matsuura S, Nagatsu T (1989) Presence of tetrahydroisoquinoline, a parkinsonism-related compound, in foods. J Chromatogr 493:347-352

Niwa T, Yoshizumi H, Tatematsu A, Matsuura S, Yoshida M, Kawachi M, Naoi M, Nagatsu T (1990) Endogenous synthesis of $N$-methyl1,2,3,4-tetrahydroisoquinoline, a precursor of $\mathrm{N}$-methylisoquinolinium ion, in the brains of primates with parkinsonism after systemic administration of 1,2,3,4-tetrahydroisoquinoline. J Chromatogr 533:145-151

Ohta S, Kohno M, Makino Y, Tachikawa O, Hirobe M (1987) Tetrahydroisoquinoline and 1-methyl-tetrahydroisoquinoline are present in the human brain: relation to Parkinson's disease. Biomed Res 8:453-456

Ortwine DF, Malone TC, Bigge CF, Drummond JT, Humblet C, Johnson G, Pinter GW (1992) Generation of $N$-methyl-Daspartate agonist and competitive antagonist pharmacophore models. Design and synthesis of phosphonoalkyl-substituted tetrahydroisoquinolines as novel antagonist. J Med Chem 35: $1345-1370$

Parsons LH, Koob GF, Weiss F (1995) Serotonin dysfunction in the nucleus accumbens of rats during withdrawal after unlimited access to intravenous cocaine. J Phramacol Exp Ther 274:1182-1191

Patsenka A, Antkiewicz-Michaluk L (2004) Inhibition of rodent brain monoamine oxidase and tyrosine hydroxylase by endogenous compounds-1,2,3,4-tetrahydroisoquinoline alkaloids. Pol J Pharmacol 56:727-734

Perry TL, Jones K, Hansen S (1988) Tetrahydroisoquinoline lacks dopaminergic nigrostriatal neurotoxicity in mice. Neurosci Lett $85: 101-104$ 
Pierce RC, Kalivas PW (1997) A circuitry model of the expression of behavioral sensitization to amphetamine-like psychostimulants. Brain Res Rev 25:192-216

Pietraszek M, Michaluk J, Romanska I, Wasik A et al (2009) 1-Methyl-1,2,3,4-tetrahydroisoquinoline antagonizes a rise in brain dopamine metabolism, glutamate release in frontal cortex and locomotor hyperactivity produced by MK-801 but not the disruptions of prepulse inhibition, and impairment of working memory in rat. Neurotox Res 16:390-407

Pitkanen A (2000) Connectivity of the rat amygdaloid complex. In: Aggleton JP (ed) The amygdala. Oxford University Press, Oxford

Popik P, Mamczarz J, Fraczek M, Widla M et al (1998) Inhibition of reinforcing effects of morphine and naloxone-precipitated opioid withdrawal by novel glycine site and uncompetitive NMDA receptor antagonists. Neuropharmacology 37:1033-1042

Pulvirenti L, Maldonado-Lopez R, Koob GF (1992) NMDA receptors in the nucleus accumbens modulate intravenous cocaine but not heroin self-administration in rat. Brain Res 594:327-330

Ritz MC, Lamb RJ, Goldberg SR, Kuhar MJ (1987) Cocaine receptors on dopamine transporters are related to self-administration of cocaine. Science 237:1219-1223

Robinson TE, Berridge KC (1993) The neural basis of drug craving: an incentive-sensitization theory of addiction. Brain Res Rev 18:247-291

Rogawski MA, Thurkauf A, Yamaguchi S, Rice KC, Jacobson AE, Mattson MV (1989) Anticonvulsant activities of 1-phenylcyclohexylamine and its conformationally restricted analog 1,1-pentamethylenetetrahydroquinoline. J Pharmacol Exp Ther 249:708-712

Rommelspacher H, Susilo R (1985) Tetrahydroisoquinolines and $\beta$-carbolines: putative natural substances in plants and mammals. Prog Drug Res 29:415-459

Rossetti ZL, Hmaidan Y, Gessa GL (1992) Marked inhibition of meso-limbic dopamine release: a common feature of ethanol, morphine, cocaine and amphetamine abstinence in rats. Eur $\mathbf{J}$ Pharmacol 221:227-234

Sánchez-Blázquez P, Boronat BA, Olmos G, García-Sevilla JA et al (2000) Activation of 1(2)-imidazoline receptors enhances supraspinal morphine analgesia in mice: a model to detect agonist and antagonist activities at these receptors. $\mathrm{Br} \mathrm{J}$ Pharmacol 130:146-152

Sandler M, Carter SB, Hunter KR, Stern GM (1973) Tetrahydroisoquinoline alkaloids: in vivo metabolites of L-dopa in man. Nature 241:439-443

Santiago M, Granero L, Machado A, Cano J (1995) Complex I inhibitor effect on the nigral and striatal release of dopamine in the presence and absence of nomifensine. Eur J Pharmacol 280:251-256

Sayre LM, Perry G, Smith MA (2008) Oxidative stress and neurotoxicity. Chem Res Toxicol 21:172-188

Schultz W (2002) Getting formal with dopamine and reward. Neuron $36: 241-263$

Schultz W (2010) Dopamine signals for reward value and risk: basic and recent data. Behav Brain Funct 6:24-38

Segal DS, Geyer MA, Schuckit MA (1981) Stimulant-induced psychosis: an evaluation of animal methods. Essays Neurochem Neuropharmacol 5:95-129

Singer TP, Ramsay RR (1995) Flavoprotein structure and mechanism 2. Monoamine oxidases: old friends hold many surprises. FASEB J 9:605-610

Smelson DA, Williams J, Ziedonis D, Sussner BD et al (2004) A double-blind placebo-controlled pilot study of risperidone for decreasing cue-elicited craving in recently withdrawn cocaine dependent patients. J Subst Abuse Treat 27:45-49

Sourkes TL (1971) Possible new metabolites mediating actions of L-dopa. Nature 229:413-414
Suzuki K, Mizuno Y, Yoshida M (1990) Effects of 1-methyl-4phenyl-1,2,3,6-tetrahydropyridine (MPTP)-like compounds on mitochondrial respiration. Adv Neurol 53:215-218

Tasaki Y, Makino Y, Ohta S, Hirobe M (1991) 1-Methyl-1,2,3,4tetrahydroisoquinoline, decreasing in 1-methyl-4-phenyl-1,2,3,6tetrahydropyridine-treated mouse, prevents parkinsonism-like behavior abnormalities. J Neurochem 57:1940-1943

Tasaki Y, Makino Y, Ohta S, Hirobe M (1993) Biosynthesis of 1-methyl-1,2,3,4-tetrahydroisoquinoline (MeTIQ), a possible antiparkinsonism agent. Adv Neurol 60:231-233

Thiffault C, Langston JW, Di Monte DA (2000) Increased striatal dopamine turnover following acute administration of rotenone to mice. Brain Res 885:283-288

Thull U, Kneubühler S, Gaillard P, Carrupt PA, Testa B, Altomare C, Carotti A, Jenner P, McNaught KS (1995) Inhibition of monoamine oxidase by isoquinoline derivatives. Qualitative and 3D-quantitative structure-activity relationships. Biochem Pharmacol 50:869-877

Trulson ME, Ulissey MJ (1987) Chronic cocaine administration decreases dopamine synthesis rate and increases $\left[{ }^{3} \mathrm{H}\right]$ spiroperidol binding in rat brain. Brain Res Bull 19:35-38

Turner AJ, Baker KM, Algeri S, Erigerio A et al (1974) Tetrahydropapaveroline: formation in vivo and vitro in rat brain. Life Sci 14:2247-2257

Ungless MA (2004) Dopamine: the salient issue. Trends Neurosci 27:702-706

Vanderschuren LJ, Kalivas PW (2000) Alteration in dopaminergic and glutamatergic transmission in the induction and expression of behavioral sensitization: a critical review of preclinical studies. Psychopharmacology 151:99-120

Vetulani J (2001) Drug addiction. Part II. Neurobiology of addiction (Review). Pol J Pharmacol 53:303-317

Vetulani J, Nalepa I, Antkiewicz-Michaluk L, Sansone M (2001) Opposite effect of simple tetrahydroisoquinolines on amphetamine- and morphine-stimulated locomotor activity in mice. J Neural Transm 108:513-526

Vetulani J, Antkiewicz-Michaluk L, Michaluk J (2003a) Modification of morphine analgesia, tolerance and abstinence by 1,2,3,4tetrahydroisoquinoline. Eur Neuropsychopharmacol 113:S29S30

Vetulani J, Antkiewicz-Michaluk L, Nalepa I, Sansone M (2003b) A possible physiological role for cerebral tetrahydroisoquinolines. Neurotox Res 5:147-155

Wąsik A, Romanska I, Antkiewicz-Michaluk L (2007) The effect of an endogenous compound 1-methyl-1,2,3,4-tetrahydroisoquinoline on morphine-induced analgesia, dependence and neurochemical changes in dopamine metabolism in rat brain structures. J Physiol Pharmacol 58:235-252

Wasik A, Romanska I, Ankiewicz-Michaluk L (2010) Important role of 3-methoxytyramine in the inhibition of cocaine sensitization by 1-methyl-1,2,3,4-tetrahydroisoquinoline: an in vivo microdialysis study. Pharmacol Rep 62:983-997

Wąsik A, Romańska I, Michaluk J, Antkiewicz-Michaluk L (2012) Comparative behavioral and neurochemical studies of R- and $\mathrm{S}$-1-methyl-1,2,3,4-tetrahydroisoquinoline stereoisomers in the rat. Pharmacol Rep 64:857-869

Weiss F, Markou A, Lorang MT, Koob GF (1992) Basal extracellular dopamine levels in the nucleus accumbens are decreased during cocaine withdrawal after unlimited-access self-administration. Brain Res 593:314-318

Wilcox KM, Rowlett JK, Paul IA, Ordway GA et al (2000) On the relationship between the dopamine transporter and the reinforcing effects of local anesthetics in rhesus monkeys: practical and theoretical concerns. Psychopharmacology 153:139-147

Yamakawa T, Ohta S (1997) Isolation of 1-methyl-1,2,3,4-tetrahydroisoquinoline-synthesizing enzyme from rat brain: a possible 
Parkinson's disease-preventing enzyme. Biochem Biophys Res Commun 236:676-681

Yamakawa T, Ohta S (1999) Biosynthesis of a parkinsonismpreventing substance, 1-methyl-1,2,3,4-tetrahydroisoquinoline, is inhibited by parkinsonism-inducing compounds in rat brain mitochondrial fraction. Neurosci Lett 259:157-160

Yamakawa T, Kotake Y, Fujitani M, Shintani H, Makino Y, Ohta S (1999) Regional distribution of parkinsonism-preventing endogenous tetrahydroisoquinoline derivatives and an endogenous parkinsonism-preventing substance-synthesizing enzyme in monkey brain. Neurosci Lett 276:68-70

Zarranz de Ysern ME, Ordoñez LA (1981) Tetrahydroisoquinolines: a review. Prog Neuropsychopharmacol 5:343-355 